\title{
Risco: a COVID-19 mobile application with contact tracing
}

\author{
Fernando López-López, Fernando Nieto-Morales, Ana Paula Huerta-Pérez, \\ Jocelyn Hazel Bolaños-Baez, Yolanda Moreno
}

Published: 30 November 2021

\begin{abstract}
The pandemic from 2019 has led us to the implementation of different protocols to reduce the number of infections per day and avoid the overwhelming of the health centers. Until 2021, the implemented methodologies to stop the continuous growth of the pandemic have greatly reduced the infections, but they are not enough for the society. Thus, we propose Risco, an application that will aid not only in the reduction of infections via the early detection of contact with infected people by providing real time analytics and contact tracing support but improve the awareness of the user location data.
\end{abstract}

\section{Keywords:}

COVID-19; Contact tracing; Mobile application; Global Position System; User-Centered design.

\section{Introduction}

Since 2020, the Covid-19 pandemic has spread around the world collapsing a variety of sectors, in special the health care areas, and has been difficult to control the propagation of it. From the commonly used methods of maintaining distance with the other individuals in public areas to the use of technologies as mobile applications to transmit awareness. This wide spreading virus led us to the search of new alternatives to stop it.

Around $45 \%$ of the global population have a smartphone, which makes it easier to realize collections of data and the distribution of these. At the same time mobile applications support audiovisual content creating a perfect opportunity to make use of them for controlling the increasing and controlling the number of infections [1].

Centering on México, the pandemic reached in January more than 2 million confirmed cases and more than 170 thousand

López-López, Fernando., Nieto-Morales, Fernando., Huerta-Perez, Ana Paula., Bolaños-Baez, Jocelyn Hazel., Moreno, Yolanda.

Universidad de las Américas Puebla

Cholula, México.

\{fernando.lopezlz, fernando.nietoms, ana.huertapz, jocelyn.bolanosbz, yolanda.moreno\}@udlap.mx confirmed deaths [2]. Each day these numbers will grow exponentially until better sanity and awareness protocols are implemented in the country.

Talking about the technological mobile devices, 84.4 million people use smartphones. This represents $\sim 66 \%$ of the Mexican population [3], nevertheless, the internet access in public places could be a problem as it is not as developed as it may be.

There are applications in México for Covid-19 as the COVID-19MX developed by Secretaría de Salud MX. The main problem of this is that it only gives information about the pandemic and recent news and requires access to Bluetooth connection of the device despite it does not support Contact Tracing [4]. Our focus with Risco is not only the creation of each detached function, but to converge them into only one application that would represent a better user experience.

The focus we have for Risco is a mobile social application with an assisted navigation system by Global Position System (GPS) that provides information about the total agglomeration in public areas. The purpose is to know if it is a good idea to visit a place or not based on this counter. It will also provide a contact tracing for the cases when a person is infected with Covid-19, which in México there are no applications with these features.

\section{Related Work}

To understand the limitations and implications to consider for the design of the application, we considered some articles to attach these problems: first, for the user's privacy and security while designing the application is crucial as all the functionality relies on them. The article [5] shows the permissions these kinds of applications require, and analyze the security measures to ensure privacy once the application is deployed using different approaches of wireless technologies, also shows the setup for Contact Tracing Applications as well as the significance of them providing guidelines to produce secure applications

Then, analyzing the existing applications with tracking functionality, they show us the potential improvement points. Besides, the manipulation of the data collected is crucial to determine the areas that may be a risk to visit or are safe places. The article [6] showed us how to improve an application where data management, transparency, privacy, and reliability are key features, and along with the previous article.

The article [7] describes how they managed the agglomeration count by using smart testing (focus only on the most possible candidate to be infected) with Bluetooth contact 
counting (the people that have installed the app will continuously try to connect to other devices to count the other possible number of people in the place) based app. The main purpose of this applied method is to only need the half of the population approximately participating in the app to work correctly while considering the existence of asymptomatic cases that will not actively participate. Applying this methodology may decrease the necessary number of needed users to create better results.

Using these recommendations will help us to improve our designs and the management of data. Similarly, we searched the actual applications that were created to decrease the Covid-19 infections. We found a review [1] where they created a summary of different apps related to Covid-19 where they collected the opinions of many users of those applications, what the users liked and the functions they would like to improve. Besides, in [4] latin american applications were compared and the article mentions a Mexican App developed by Secretaría de Salud MX, which gave us a good scene of how to focus our application on Mexico. Thus, we can afford a better user-centered design for the desired application [8].

\section{Methodology}

To achieve a good design for the application, firstly we had a brainstorming session, in which many ideas came up and others were discarded. The ideas were shared with our professors, that gave us feedback on which parts could be improved or modified. Once the ideas were established, we searched about the Mexican context, as most of the articles found are from other countries, and based on the information found we decided that the requirements for the application are the following:

- Multiplatform (iOS/Android)

- $\quad$ Easy to use

- Clean User Interface

- Consistency with the designs and interactions

Then, the next steps followed are the design of the applications and its functionalities, and the Evaluation.

\section{Design}

The research to create the prototype was done based on the main problems to have an approach to a User Centered Design. We identify the necessary functions that must be implemented:

- Agglomeration measure: Check the total of people in public areas.

- Level of risk: Based on the previous function and the prevention measures given by the health organizations, check the probability of the user to be infected.

- $\quad$ Sanity and Safe locations: Set the closer sanity stations and safe places (that maintain the health protocols for individual space) in the map.

- COVID prevention: If a user is infected, the user might have the chance of advice through the application to realize a contact tracing.

Demonstrating the relevance of these, we use a storyboard (Figure 1) of the common user in which the app is focused. The main problem is the lack of information of the users from the public areas: The sanity protocols that are being used, the total number of people in the place, the risk of being infected in specific zones. For the user, maintaining a constant acknowledgement of data of public zones can make the difference.

\footnotetext{
${ }^{1} \underline{\mathrm{https}} / / / \mathrm{miro} . c 0 \mathrm{~m} / \mathrm{app} /$ board/o9J_lxned_o=/
}

To grant a well-structured application, we made four phases: Context of Use (previously mentioned in the article), Design, Evaluation and Discussion.

For the design the first step was to determine the motivations and requirements for the application ${ }^{1}$.

- Motivations: The needs of users to know which places are safe to visit and the best hours to shopping, guaranteeing safety for the user and the surrounding people.

- Requirements: Based on the functions previously mentioned and the related work, location tracking, user account, access to map, online connection (Bluetooth/Wi-Fi), and keeping the application running at least as a background process, are crucial for a correct functionality.

The desired design of the application is that the mentioned functions will be applied over an GPS system navigation. For that, the process in which the app will work is: Select the place you want to visit and show the distance from the actual location of the user to the place. The app must show the total of people it has counted on the selected place and, in the case that there is a possible risk of getting infected (due to the actual agglomeration is over the average of the sanity protocols or a contact tracing process showed that an infected was located there) and advise the user if it is safe or not. It will also provide the information of any sanity place near the selected location to maintain informed the user of where it is possible to get tools and objects to secure its health in the case of any emergency in the travel.

We take into account the possibility that individuals in the population will not want to share their actual health state due to any personal reason. Thus, we will constantly motivate the users to participate in the app by sending notifications before and after each travel. Due to the importance of the privacy of each person, we cannot afford the intrusion inside the information of an individual that does not desire to share their data. Furthermore, any collected info will be eliminated after some established time (30 days since collection) to maintain the privacy of the users.

For designing scenarios of the features mentioned, we designed personas considering common situations as the one seen in the Figure 1. We analyze different perspectives and not only 
those who will be the ideal users of the app. We put the description of all personas into the design board as well.

Considering the mentioned functionalities, the design team created a low-fidelity prototype interface using the Balsamiq ${ }^{2}$ software, some examples of these can be seen in the Figure 2:

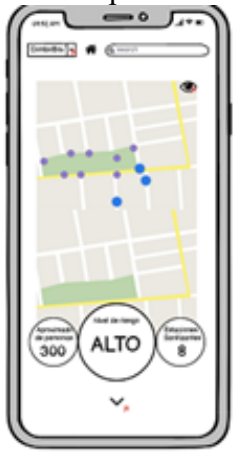

a)

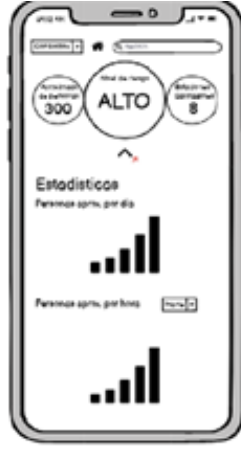

b)
Figure 2: Wireframes from the application. a) Main page of the application contains a map view of the place that you visit to visualize all the sanity points in the area, the quantity of people and the level of risk of the area. b) Page with explicit information of the selected place, these includes the total number of people in the place, Risk of getting infected \& Total number of sanity points in the area.

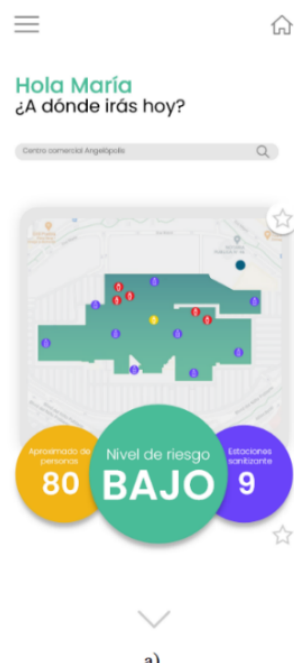

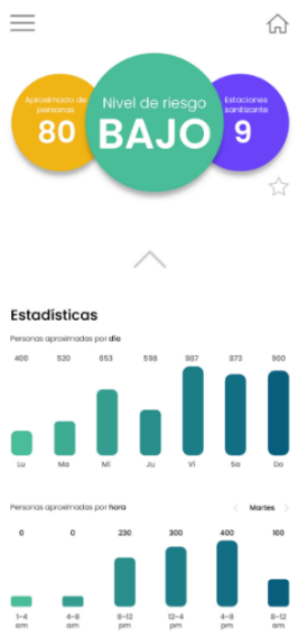

b)
Figure 3: High Fidelity mockup of the application. a) Main page. b) Analytics of the area.

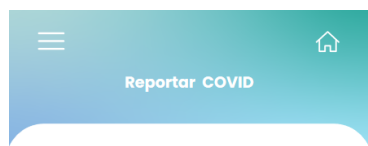

¿Has sido diagnosticado con
COVID estos ultimos 10 dias?
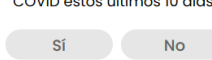

¿Qué día recibiste tu diagnóstico?
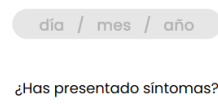

Si No

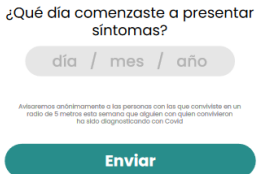

Figure 4: Form for new covid infected that triggers the contact tracing notifications.

Once the low-fidelity prototype was finished and verified by the team, we created a high-fidelity prototype. The details about each zone as well as the statistics are displayed accordingly to the user's preferences (Figure 3). Besides, a form was included, where users can fill it anonymously if they have covid (Figure 4). Finally, a help section (Figure 5.a) was included as well as a simple user profile section (Figure 5.b), where the user can learn the meaning of the symbology and the last places they have visited respectively.

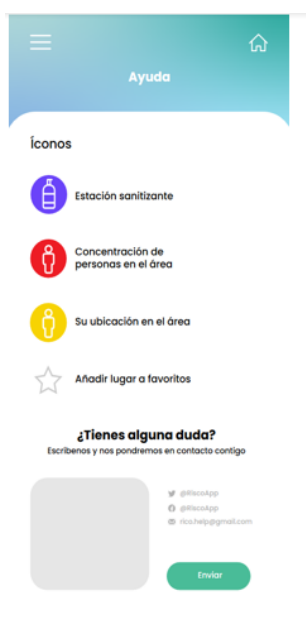

a)

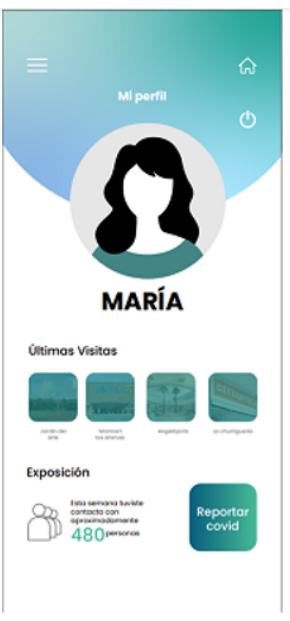

b)
Figure 5: High Fidelity mockup of the application. a) Help section. b) User profile section

\section{Evaluation}

Based on the high-fidelity prototype, we conducted a usability test. The tasks to be done by the testing user are: Create an account and log in; select a location, see the risk in the area and consult the schedule to observe the best hour to visit the place (Figure 3.a); supposing that the user was infected, move to the

\footnotetext{
${ }^{2}$ https://balsamiq.cloud/sn0j8zb/phhmle5
} 
Covid report form, complete it and send it (Figure 4); supposing that the user does not understand a symbol in the map, move to the help section and see the meaning of it (Figure 5.a); check the total number of people that has been in contact with you (Figure 5.b).

For the usability testers we consider the participants: four males and four females, with a range of age from 19 to 51 years. The selection of these is based on the target audience mentioned in the Introduction. We give them the prototype in a cellphone, and we told them to realize the tasks.

The results of the usability test are that the flow of the application was good and simple enough. Most of the people that tested the application could use it without problems, but the positioning and functionalities of some icons was confusing. After refining some details on the design with the results gathered and adding features as a short tutorial of the functionalities the application provide, the final design is as following: on Figure 3.a, the main page can be seen, with the level of risk being displayed on the circle in the center, on the right the number of sanitizing stations and on the left the number of people in the area; on Figure 3.b, the statistics of the average number of people by day that visit the area, and also the average number of people on the selected day by 4 hours intervals. At last, for the contact tracing feature (Figure 4), a form is required for those who got covid, this to send a notification for the people that were close to him during the past days, which can help to maintain an early quarantine, the profile (Figure 5.a) and help (Figure 5.b) sections had no modifications.

\section{Discussion}

As a result of the usability test, we obtained that in general the application is easy to use. In the first 3 tasks any of the users needed help and realized them easily. The task of obtaining the meaning of the symbols presented two obstructions in its realization by two testers, they needed guidance to find the symbology. Finally, the las task of finding the total contacts the user had had more conflicts than the others. Three users needed guidance to find the number, but the difference of time to realize this activity with the others is small. From the usability tests we can mention the application results easy to understand.

Risco provides a helpful way to avoid concurred places by providing visual feedback and analytics of the desired place. Also, the application aims to be used by a big quantity of people as it is important for the analytics and contact tracing features. The implementation can be quite difficult as the research made shows the importance of privacy and how some people avoid sharing their location in real time. Also, the lack of importance and awareness about the covid 19 in Mexico is challenging, as well as the scarce points of public Wi-Fi.

To conclude, Risco showed a great aid to the user acknowledge of their information, and it can also be improved.
There are other functions that can be implemented to create a better awareness such as a News section to know the actual information of the pandemic and the actual color Traffic light in the user zone, a Guide section for the infected people of what they should do, and more. Risco can provide many functions for the community.

\section{References}

[1] Islam, M. N., Islam, I., Munim, K. M., \& Islam, A. N. (2020). A review on the mobile applications developed for COVID-19: an exploratory analysis. IEEE Access, 8, 145601-145610.

[2] Morgenstern-Kaplan, D., Rocha-Haro, A., CanalesAlbarrán, S. J., Núñez-García, E., \& León-Mayorga, Y. (2021). An App-Based Telemedicine Program for Primary Care and Specialist Video Consultations During the COVID-19 Pandemic in Mexico. Telemedicine and $e$ Health.

[3] Instituto Federal de Telecomunicaciones. (2020, 17 febrero). En México hay 80.6 millones de usuarios de internet y 86.5 millones de usuarios de teléfonos celulares: ENDUTIH 2019. February, 17. Instituto Federal de Telecomunicaciones - IFT. IFT. http://www.ift.org.mx/comunicacion-ymedios/comunicados-ift/es/en-mexico-hay-806-millonesde-usuarios-de-internet-y-865-millones-de-usuarios-de$\underline{\text { telefonos-celulares }}$

[4] Fernández Sosa, J. F., Aguirre, V., Delía, L. N., Thomas, P. J., Corbalán, L. C., \& Pesado, P. M. (2020). COVID-19: un análisis comparativo de Apps. In XXVI Congreso Argentino de Ciencias de la Computación (CACIC)(Modalidad virtual, 5 al 9 de octubre de 2020).

[5] Azad, M. A., Arshad, J., Akmal, S. M. A., Riaz, F., Abdullah, S., Imran, M., \& Ahmad, F. (2020). A first look at privacy analysis of COVID-19 contact tracing mobile applications. IEEE Internet of Things Journal.

[6] Gupta, R., Bedi, M., Goyal, P., Wadhera, S., \& Verma, V. (2020). Analysis of Covid-19 tracking tool in India: Case study of Aarogya Setu mobile application. Digital Government: Research and Practice, 1(4), 1-8.

[7] Hossein, G., Arnoldini , M., Jenny, D., Hardt, W.-D., \& Jenny , P. (2020). STeCC: Smart Testing with Contact Counting Enhances Covid-19 Mitigation by Bluetooth App Based Contact Tracing. Switzerland: medRxiv.

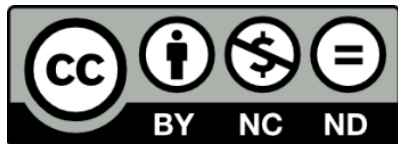

C 2021 by the authors. This work is licensed under the Creative Commons AttributionNonCommercial-NoDerivatives 4.0 International License. To view a copy of this license, visit http://creativecommons.org/licenses/by-nc-nd/4.0/ or send a letter to Creative Commons, PO Box 1866, Mountain View, CA 94042, USA. 